\title{
Discrete Contour Extraction from Reference Curvature Function
}

\author{
H.G. Nguyen ${ }^{1}$, B. Kerautret ${ }^{1}$, P. Desbarats ${ }^{2}$, and J.-0. Lachaud ${ }^{3}$ * \\ 1 LORIA, Nancy-University - IUT de Saint Dié des Vosges \\ 54506 Vandœuvre -lès-Nancy Cedex, France \\ \{kerautre, Huugiao. Nguyen\}@loria.fr \\ 2 LaBRI, University of Bordeaux 1 \\ 351, cours de la Libration 33405 Talence, France \\ desbarats@labri.fr \\ 3 LAMA, University of Savoie \\ 73376 Le Bourget du Lac \\ jacques-olivier.lachaud@univ-savoie.fr
}

\begin{abstract}
A robust discrete curvature estimator was recently proposed by Kerautret et al. [1]. In this paper, we exploit the precision and stability of this estimator in order to define a contour extraction method for analysing geometric features. We propose to use a reference curvature function for extracting the frontier of a shape in a gray level image. The frontier extraction is done by using both geometric information represented by the reference curvature and gradient information contained in the source image. The application of this work is done in a medical application.
\end{abstract}

\section{Introduction}

Extracting geometric characteristics of digital objects is an important step in the field of image analysis. The application domain is large like in medical imaging or in archaeology. Area, perimeter or curvature estimator can be used to characterize digital objects of interest. Obtaining precise

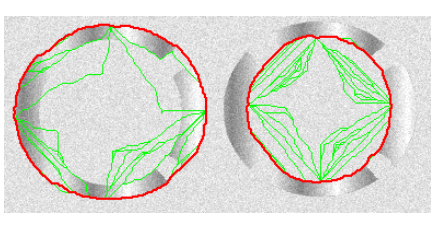
geometric measure is not always a simple task since it depends both on the geometric estimators and on the technique providing the digital contour. The main idea of this work is to propose a method for recovering contour shape by directly extracting the frontier from geometric constraints. The geometric constraints will be defined mainly by the curvature function allowing to obtain a solution from user initialisation even when several contours can be found (see figure above).

Numbers of different approaches were proposed throughout the literature dealing with image segmentation. Generally the image components are extracted from a contour or region approach which exploits additional and a priori information. This information can be defined for example from smoothness constraints, geometric model of reference, or user interaction. A well known example

* Bertrand Kerautret was partially funded by a BQR project of Nancy University. 
of Energy-minimizing approach including smoothness constraints are snakes or active contours [2-4]. Another approach called Active Shape Model (ASM) [5] used a parametric model based on statistical information. They used statistical information to define parameters. Another well known technique for discrete interactive segmentation is the intelligent scissors [6]. This technique helps the user to define contours by computing image gradient and computing minimal path from user defined points. It was frequently used in medical application for shapes measures as in $[7,8]$. Other more recent techniques exploit this idea (lazy snaping [9], enhanced lane [10] or grabcut [11]).

Exploiting directly the quantitative curvature evolution has not yet been applied to give a priori constraints for shape/contour extraction. Even if recently Schoenemann and Cremers introduced curvature to determine globally an optimal solution [12], their approach does not use quantitatively the curvature as a reference model. Another recent work from Färber et al. proposed a Live-wire based segmentation approach to associate corresponding image structures. In that case, the curvature was only used as a parameter for the contour association [7].

Our main objective is to use quantitative shape description from the curvature values in order to extract contours and in a semi-automatics process. The curvature will there guide the segmentation process. Our approach is based on the Global Min-Curvature (GMC) estimator introduced in [1] and is using the shortest path algorithm defined on the live-wire method. The main advantage of the GMC estimator is its noise robustness and the stability which allows to extract directly geometric information as for example local maxima/minima curvature points.

The paper is organized as follows: some main notions of previous works on curvature estimators are reviewed in the following section. Then section 3 introduces the proposed method used to construct and select a list of candidates for the contour between two reference points. Finally, section 4 is devoted to the medical application which validates our approach by some experiments on several types of normal and pathological MR images of human brain.

\section{Global Min-Curvature estimator (GMC)}

The main idea of this estimator is first to take into account all the shapes having the same digitization and to select the more probable shape defined as thus which minimize the squared curvature. This selection is done with a global optimisation process. By this way we can expect to obtain a precise estimator even with low resolution shapes. The second idea is to obtain precise results even with non perfect digitization processes inducing noisy contours.

The selection of the more probable shape is defined from geometric constraints extracted from the tangential cover [13]. The notion of tangential cover is illustrated on the figure 1(a) which shows all the maximal segments of a discrete shape. From each maximal segments the minimal and maximal possible values of the tangent (see Fig. 1(b)) are used as constraints to estimate the shape minimizing the squared curvature. The optimisation process is then 


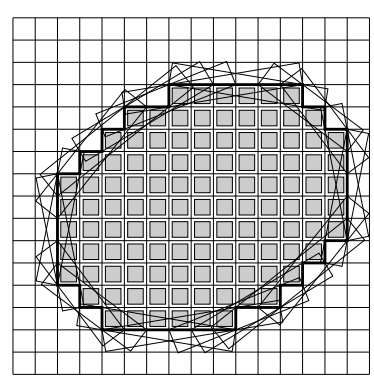

(a)

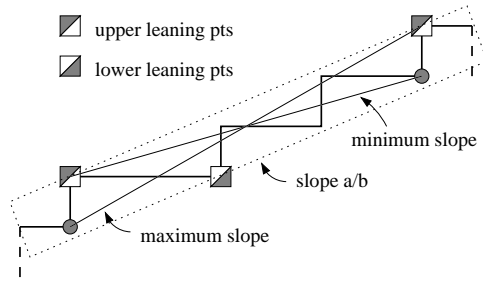

(b)

Fig. 1. (a): tangential cover of the boundary of a digitized shape, where each maximal segment is drawn as a black bounding box aligned with its slope. (b): slope of a maximal segment and estimation of maximal and minimal slopes with leaning points.

achieved with a relaxation approach. To obtain robustness to noise the maximal discrete segments from the tangential cover are replaced by the blurred maximal segments [14] which allow to take into account the amount of noise. Note that a parameter $\nu$ permits to control the sensibility to noise. More details about the GMC estimator are presented in [1].

The resulting curvature estimator gives precise and stable results. As illustrated in Fig. 2, the precision obtained on a circle shows precise results compared for example to the estimator based on the osculating circles [15]. The column (c) of Fig. 2 illustrates the extraction of local minima/maxima on a generated font obtained at $300 \mathrm{dpi}$. We can see that the GMC estimator gives good local minima/maxima compared to the CC estimator. The bad results of the CC estimator are due to the lack of stability since numerous oscillations appear even with large resolution. Note that the local minima/maxima values were simply extracted from the curvature graph by a simple value quantification at a given precision. Contrarily to other curvature estimators, no post-processing is needed in order to exploit values and thus, there is no risk of degradation of the extracted geometric informations.

Experiments and comparisons were applied on noisy shapes with different estimators. The GMC estimator was compared with the "blurred" version of CC estimator proposed by Nguyen and Debled-Rennesson [16] (called NDC estimator). As in the smooth case, the GMC estimator always shows more precision and stability than the NDC estimator. Details and comparisons can be found in previous work [1].

In the following our main idea is to exploit the stability and the precision of the GMC estimator in order to define a robust new approach for shape segmentation. Moreover the resistance to noise with the choice of the parameters associated to the width used for the analysis can contribute to new perspectives. 


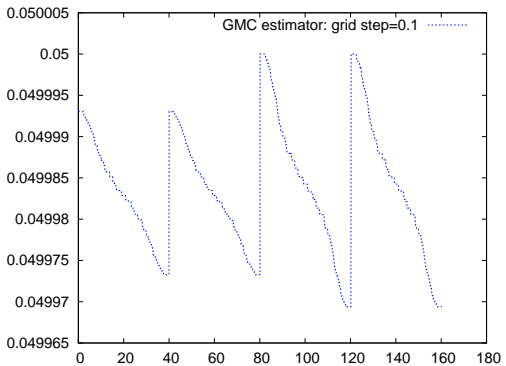

(a)

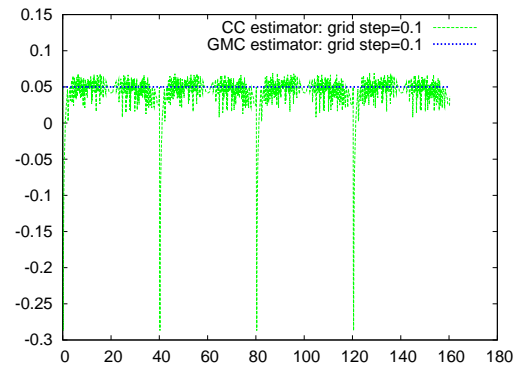

(b)

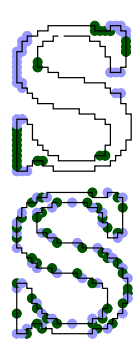

(c)

Fig. 2. Results and comparisons with the $\mathrm{CC}$ estimator on a circle of radius 20 with grid step $=0.1$ (graph (a) and (b)). Extraction of the local maxima $/ \mathrm{minima}$ with GMC (top of column (c)) and CC estimators (bottom of column (c)) . Dark (resp. light) areas represent local minima (resp. maxima) (green (resp. blue)).

\section{Contour Selection from Curvature Information}

We define by $P_{s}$ and $P_{e}$ the two reference points which need to be initialized by the user in respect to the reference curvature. The reference geometric informations of segment $P_{s} P_{e}$ are defined as constant. From the initial reference shape, we compute the mean curvature value $C_{r e f}$ of $P_{s} P_{e}$ by using GMC estimator with a specific width $\nu$ defined according the amount of noise contained in the image. We estimate a possible distance for a change of this curvature by two values : $C_{\max }$ and $C_{\min }$. A fourth parameter was defined by the admissible ratio error of the perimeter $E r r_{p e r i}$ of $P_{s} P_{e}$.

\subsection{Construction of the list of the potential candidates}

Let us construct a shortest path map from a target point to all other points in the image. The cumulative cost of a path from a target pixel was proposed by Mortensen et al. in 1995 [6]. The local cost of the image pixels is defined from the different edge features. In this work, the Sobel operator was used to compute the gradient magnitude $f_{G}$ and the gradient direction $f_{D}$ of the source image. The information of laplacian zero-crossing $f_{Z}$ and Edge Pixel Value $f_{I}$ are considered as the important components of the cost of the pixel. To increase noise robustness, we chose the following function to determine the cost from a point $\mathrm{p}$ to a neighboring point $\mathrm{q}$ :

$$
l(p, q)=0.7 * f_{G}+0.1 * f_{D}+0.1 * f_{Z}+0.1 * f_{I}
$$

Note that the coefficients of $f_{G}, f_{D}, d_{Z}$ and $f_{I}$ are determined empirically as mentioned by the author in [6]. After computing the local cost of the pixels, we use the shortest path algorithm [17] to construct the relation map from a reference point to all other points of the image. 
We denote the shortest path from $P_{s}$ and $P_{e}$ by $S\left(P_{s}, P_{e}\right)$ which is easy to extract from the shortest path map of $P_{s}$. The shortest path getting from point $P_{s}$ to $P_{e}$ through point $p_{k}$ is denoted by $S_{k}: S_{k}=\left\{S\left(P_{s}, p_{k}\right), S\left(p_{k}, P_{e}\right)\right\}$.

In order to recover the closest contour to the ideal reference solution, we need to obtain a list of potential candidates. For this purpose, we propose a method to construct a list of potential candidates $A$ associated to the segment $P_{s} P_{e}$. We denote by $P_{s}^{\prime}$ and $P_{e}^{\prime}$ the image of $P_{s}$ and $P_{e}$ obtained by a rotation of $\theta$ centered at $M$ defined as the center of $P_{s} P_{e}$. The list of potential candidates $A^{\theta}$ associated to the angle $\theta$ is defined by:

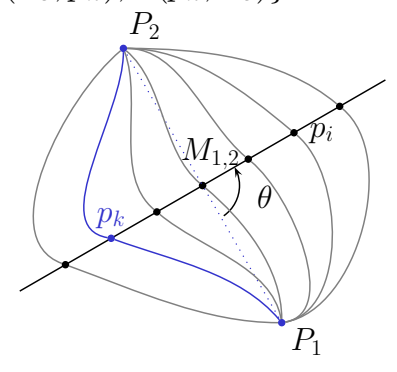

$$
A^{\theta}=\left\{Q_{k} \mid Q_{k} \in \mu^{\prime}<a^{\prime} x+b^{\prime} y+c^{\prime}<\mu^{\prime}+\omega^{\prime} \text { and } \overline{Q_{k} M}>\overline{Q_{k+1} M}\right\} ;
$$

with: $a^{\prime}, b^{\prime}, c^{\prime}$ associated to the straight segment defined by $\left(P_{s}^{\prime}, P_{e}^{\prime}\right)$. To increase the probability to obtain the best candidate list, we use three values of $\theta$ : $\frac{\pi}{4}, \frac{\pi}{2}$ and $\frac{3 \pi}{4}$. The total list of candidate points $A$ is then $A=A^{\frac{\pi}{4}} \cup A^{\frac{\pi}{2}} \cup A^{\frac{3 \pi}{4}}$. The construction of the potential candidates is illustrated on the floating figure on the right.

\subsection{Selection through the possible candidates}

From the list of possible potential candidates, we propose the following three selection steps in order to select the best contour according the geometric and photometric image constraints.

\section{- Selection based on local minima value of energy}

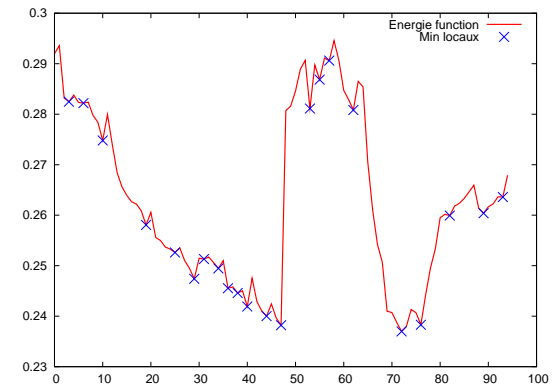

(a)

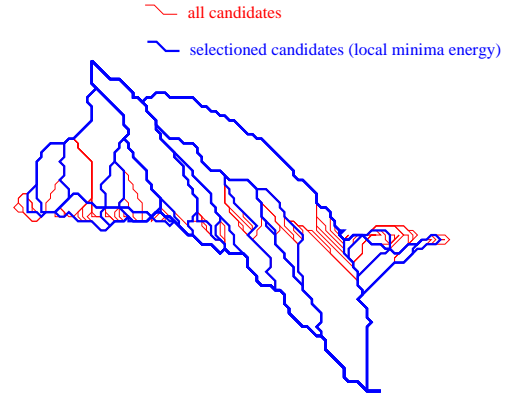

(b)

Fig. 3. Illustration of the energy curve associated to each candidate (computed on the segment $\left.P_{s}, P_{e}\right)$. The candidates with local minimal energy are represented by a cross (a). The contours associated to each candidate are is represented in figure (b). The contour with local minimal energy are represented with large line width (in dark). 
The first selection of candidates is based on its energy value in order to retain only the significant points and to reduce the number of candidates. We compute the energy $E_{k}$ defined for each point $p_{k} \in A$ by:

$$
E_{k}=G\left(S_{k}\right)=G\left(S\left(P_{s}, p_{k}\right)\right)+G\left(S\left(p_{k}, P_{e}\right)\right)
$$

The function $G$ gives the average value of a path coast and can be defined as: $\frac{\sum_{i=0, n} g\left(x_{i}\right)}{n}$ with $g(x)$ giving the coast function of the discrete point $x$ in the shortest path algorithm and $n$ represents the number of contour points.

Then, the new list of candidates $A_{1}$ is selected by minimizing locally the energy $E_{k}$ of all the candidates of the list $A$.

\section{- Selection according length constraint}

After using the information of local energy to remove the weak candidates, we can bypass the particular ambiguous cases of candidate by using the length constraint. It is particularly relevant because in some particular cases there can exist several contour solutions with the same curvature value.

The following figure on the right illustrates such a particular case. To avoid this ambiguity, we estimate the length $L_{k}$ defined for each candidate $p_{k} \in A_{1}$ of $P_{s} P_{e}$ as follows: $\sum d\left(x_{i}, x_{i+1}\right)$ where $d\left(x_{i}, x_{i+1}\right)$ gives the distance from $x_{i}$ to the next neighbouring in the contour candidate $x_{i+1}$. In order to get a significant value of length for a candidate, we must detect special

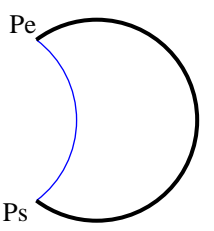
configurations where the contours overlap. From the information of length $L_{k}$ of contour, we select the new list of candidates $A_{2}$ by the following constraint:

$$
A_{2}=\left\{p_{k} \in A_{1} \mid \frac{\left|L_{k}-L_{r e f}\right|}{L_{r e f}} \leq E r r_{p e r i}\right\}
$$

Fig. 4 shows an example of candidate selection obtained from the initial list (a) representing all the initial candidates.

\section{- Adding curvature constraints :}

When the second selection of candidates is done, we use GMC estimator to measure the curvature value $C_{k}^{i}$ of the points $i$ in the candidate list $p_{k} \in A_{2}$ of $P_{s} P_{e}$. Since the curvature estimator is stable enough, the curvature values are useful to evaluate these remaining candidates and to select the best candidate of an interval $P_{s} P_{e}$.

The average value of curvatures $C_{k}^{*}$ of candidate $\mathrm{k}$ is defined as $C_{k}^{*}=$ $\frac{\sum_{i=0, n}\left(C_{k}^{i}\right)}{n}$ where $\mathrm{n}$ is the number of points for this candidate. The smallest list of candidate $A_{3}$ will be selected by a constraint to limit the range of curvature :

$$
A_{3}=\left\{p_{k} \in A_{2} \mid C_{M i n} \leq C_{k}^{*} \leq C_{\text {Max }}\right\} ;
$$

We calculate the quadratic error between the average curvature of candidate $p_{k} \in A_{3}$ and the curvature of its reference which is defined as : $E r r_{\text {quad }}=$ $\left(C_{k}^{*}-C_{r e f}\right)^{2}$. The minimal value of $E r r_{\text {quad }}$ is used to determine the best contour of interval $P_{s} P_{e}$. 


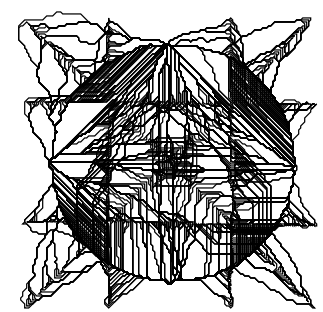

(a)

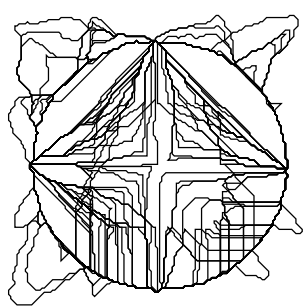

(b)

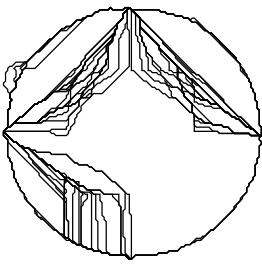

(c) $\operatorname{Err}_{\text {peri }}<10 \%$

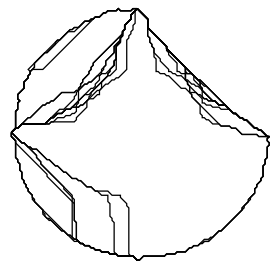

(d) $\operatorname{Err}_{\text {peri }}<5 \%$

Fig. 4. Illustration of the candidates obtained on a noisy circle $(R=61)$. Four points on the top, bottom, left, right of the circle are used to build the list of four segments $P_{s} P_{e}$. Image (a) represents all potential candidates. Image (b) shows the contours of (a) which have the minimal local energy and without the overlapping contours. The two images (c) and (d) show the selected candidates which satisfy the length constraint Err $r_{\text {peri }}$.

\section{Experiments and application}

Our first experiment (Fig. 5) illustrates the results obtained on a damaged circular test shape. The reconstruction was obtained from a constant curvature value of 0.0163 and the length error constraint was set to $20 \%$. All of candidates are represented in light blue. The contours were well recovered in white, even with a non precise initialisation (c), the global contour is correct. Note that initial points could be easily adjusted in a post processing step. The illustration images of the introduction shows the results on noisy images obtained with several reference curvature values.

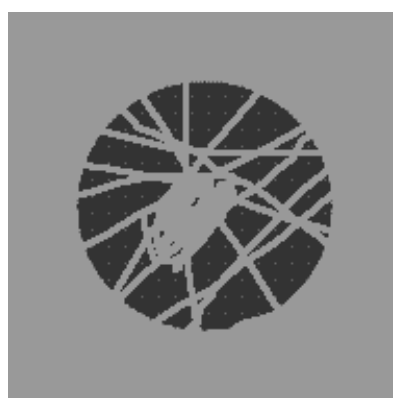

(a)

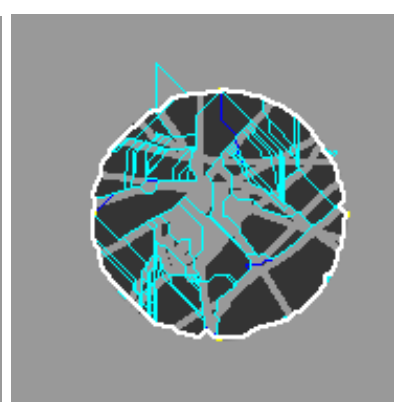

(b)

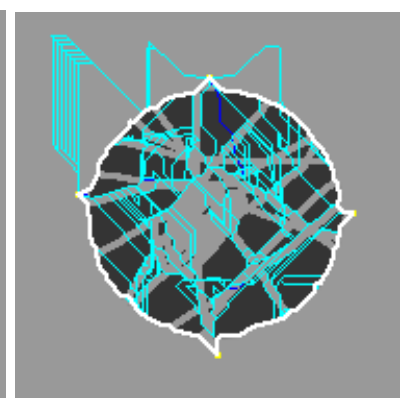

(c)

Fig. 5. Results obtained on a test image (a). The curvature of reference was set to 0.0163. The lines in light blue represent all the candidates for the segmentation and the white contour is the result of the segmentation. Image (c) shows the result with a non perfect initialisation. 
The main objective of our approach is to use quantitative description of the shape from the curvature values in order to extract contours. An application of the use of these curvature values is to make an early diagnostic of a particular Parkinson's disease syndrome. Progressive Supranuclear Palsy (PSP) is an atypical degenerative parkinsonian syndrome [18]. It leads to postural instability with falls up to subcortical dementia. Its diagnostic can be established by clinicians using conventional MRI exams. However, the differentiation between different parkinsonian syndromes is not easy and often leads to erroneous diagnostics [19]. Furthermore this differentiation is only qualitative and very difficult to achieve in the earlier stages of the syndrome, when medical treatment is still able to slow down the degenerative process. It is thus important to develop a quantitative method to make an earlier diagnostic for this particular syndrome. PSP can be spotted in MR images as an atrophy of the mid-brain part of the brain stem. This atrophy leads to a curvature changing on the upper surface of the mid-brain.

The images used in the following experimentation were undertaken on a Philips Medical Systems 1.5 Tesla Intera MR acquisition system using a T1 SE (Spin Echo) scanning sequence. Fig. 6 shows the result obtained on two cases extracted from MR images which were used to diagnose PSP. The results shown on the first row were obtained from a set of reference values $\lambda_{1}\left(C_{\text {ref }}=\right.$ $0.0549, E r r_{\text {peri }}=1, C_{\text {Min }}=0.05, C_{\text {Max }}=0.1$ ). The contours were correctly extracted with the curvature value $\boldsymbol{C 1}=\mathbf{0 . 0 5 4 0 3 5 4}$. Since the curvature result of the healthy patient is close with the $C_{r e f}$ of $\lambda_{1}$, we continued to used the $\lambda_{1}$ for the comparison with the others MR images. The image in the second row seems to be a suspected case of PSP. The curvature value of the best contour was $C 2=0.0605799$. The distance between the obtained curvature value to the reference value $C_{r e f}$ is used to discriminate the images and to diagnose pathological cases. Our last experiment for this approach is depicted in the third row of Fig. 6 which shows the contour extraction of three parts of the brain stem of MR image. Here, we must use three sets of reference value. Note that the curvature sign of (c) is negative since we consider the initialisation order. The tabular in figure Fig. 6(j) shows timing measures with the number of candidates needed by the optimization process, where $N_{\text {all }}$ and $N_{\text {est }}$ are respectively the total number of candidates and the number of candidates which have been evaluated. This measures were obtained on a $1.5 \mathrm{GHz}$ Intel Celeron $M$ processor with MR images of resolution $300 \times 300$ pixels.

\section{Conclusion}

The main contribution of this work was a proposition of a new simple method to extract image contours by using predefined curvature informations. The extraction was based on a robust curvature estimator and on the construction of shortest paths from image gradient informations. The application to medical application appears promising and future work will deal with the medical vali- 


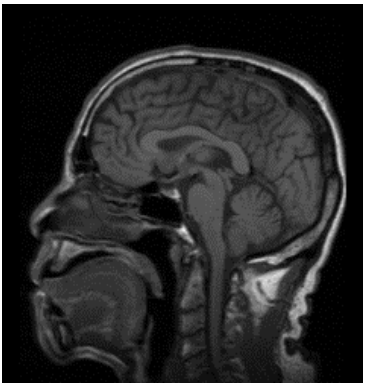

(a) patient (1)

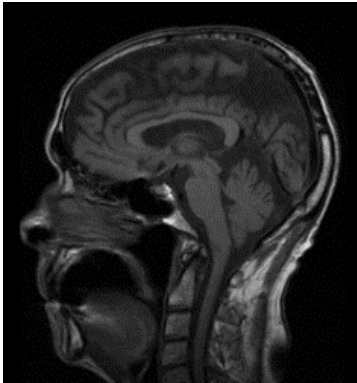

(d) patient (2)

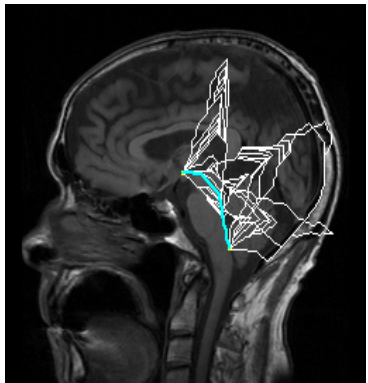

(b) all candidates

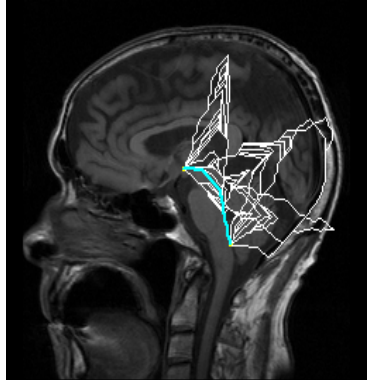

(e) all candidates

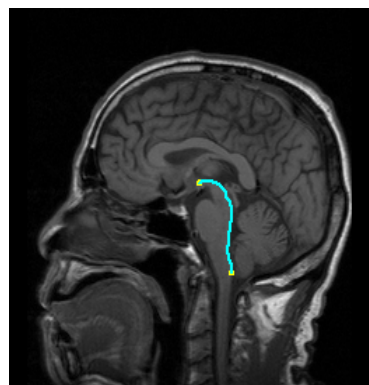

(c) curvature value $\mathrm{C}=0.0540354$

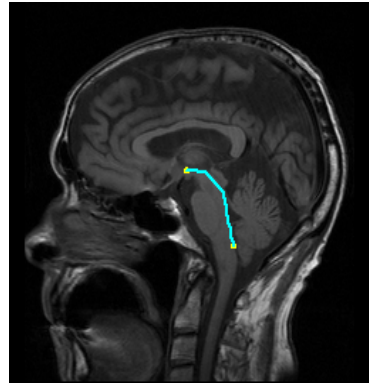

(f) curvature value $\mathrm{C}=0.0605799$

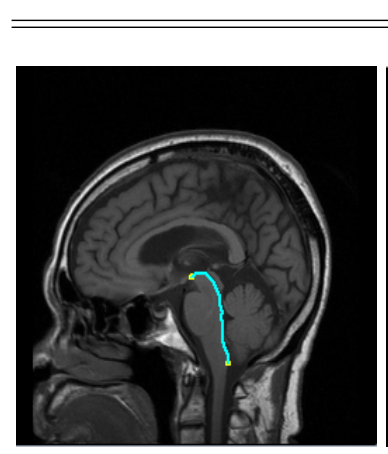

(g)

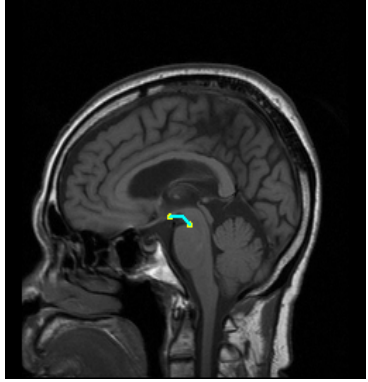

(h)

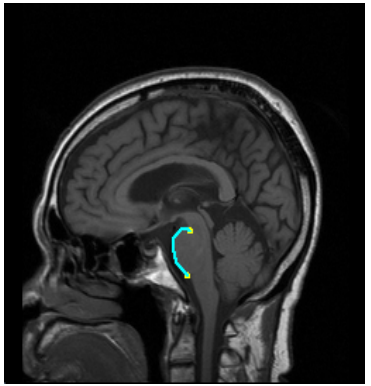

(i)

\begin{tabular}{ccccccccc}
\hline \hline Image & $C_{\text {ref }}$ & Err $_{\text {peri }}$ & $C_{M a x}$ & $C_{\text {Min }}$ & $C_{\text {Result }}$ & $N_{\text {all }}$ & $N_{\text {est }}$ & time $(\mathrm{ms})$ \\
\hline g1 & 0.0549 & 1 & 0.05 & 0.1 & $\mathbf{0 . 0 6 0 1 7 3 6}$ & 128 & 80 & 3153 \\
g2 & -0.15 & 0.5 & -0.1 & -0.2 & $\mathbf{- 0 . 1 8 6 6 5 9}$ & 25 & 19 & 170 \\
g3 & 0.087 & 1 & 0.06 & 0.1 & $\mathbf{0 . 0 8 4 5 2 5 5}$ & 70 & 32 & 671
\end{tabular}

(j)

Fig. 6. Results and comparisons on two MR images (first and second row). Images (b,e) illustrate all the candidates used for the solution selection and the selected contours are shown in $(\mathrm{c}, \mathrm{f})$. The third row show other contours extraction obtained with other curvature parameters. Tabular $(\mathrm{j})$ shows parameter values and time measures. 
dation in the context of the parkinson's atypical disease. Another future work will deal with the extension of this approach to 3D images.

\section{References}

1. Kerautret, B., Lachaud, J.O.: Robust estimation of curvature along digital contours with global optimization. In: Proc. of the Int Conf on DGCI. Volume 4992 of LNCS., Lyon, Springer (2008) 334-345

2. Kass, M., Witkin, A., Terzopoulos, D.: Snakes: Active contour models. International Journal of Computer Vision 1(4) (1988) 321-331

3. Caselles, V., Kimmel, R., Sapiro, G.: Geodesic active contours. In: Proc of Int. Conf. ICCV. (1995) 694-699

4. Lachaud, J.O., Vialard, A.: Discrete deformable boundaries for image segmentation. Research report 1244-00, LaBRI, Talence, France (2000)

5. Cootes, T., Taylor, C., Cooper, D., Graham, J.: Active shape models their training and application. Computer Vision and Image Undestranding (1995) 38-59

6. Mortensen, E.N., Barrett, W.A.: Intelligent scissors for image composition. In: Proc. of ACM SIGGRAPH 95:. (1995) 191-198

7. Farber, M., Ehrhardt, J., Handels, H.: Live-wire-based segmentation using similarities between corresponding image structures. Computerized Medical Imaging and Graphics 31 (2007) 549-560

8. AJ Gougoutas a, d.A.W., Borthakur, A., Shapiro, E., Udupa, J.: Cartilage volume quantification via live wire segmentation. Acad Radiol 11(12) (2004) 1389-95

9. Li, Y., Sun, J., Tang, C.K., Shum, H.Y.: Lazy snapping. Proc. of ACM SIGGRAPH 23(3) (2004) 303-308

10. Kang, H.W., Shin, S.Y.: Enhanced lane: interactive image segmentation by incremental path map construction. Graphical Models 64 (2003) 282-303

11. Rother, C., Kolmogorov, V., Blake, A.: "grabcut": interactive foreground extraction using iterated graph cuts. Proc. of ACM SIGGRAPH 23(3) (2004) 309-314

12. Schoenemann, T., Cremers, D.: Introducing curvature into globally optimal image segmentation:minimum ratio cycles on product graphs. In: Proc. of Int. Conf. on Comp. Vis. (2007) 1-6 Rio de Janeiro.

13. Feschet, F., Tougne, L.: Optimal time computation of the tangent of a discrete curve: Application to the curvature. In: Proc of 8th Int. Conf. DGCI'99. Number 1568 in LNCS, Springer Verlag (1999) 31-40

14. Debled-Rennesson, I., Feschet, F., Rouyer-Degli, J.: Optimal blurred segments decomposition of noisy shapes in linear times. Computers and Graphics (2006)

15. Coeurjolly, D.: Algorithmique et géométrie pour la caractérisation des courbes et des surfaces. PhD thesis, Université Lyon 2 (2002)

16. Nguyen, T., Debled-Rennesson, I.: Curvature estimation in noisy curves. In: Proc. of Int Conf. CAIP. Volume 4673 of LNCS., Springer (2007) 474-481

17. Dijkstra, E.: A note on two problems in connexion with graphs. Numerische Mathematik (1959) 269-271

18. Savoiardo, M.: Differential diagnosis of parkinson's disease and atypical parkinsonian disorders by mangnetic resonance imaging. Neurol Sci 24 (2003) 35-37

19. Schrag, A., Good, C., Miszriel, K., Morris, H., Mathias, C., Lees, A., Quinn, N.: Differentiation of atypical parkinsonian syndromes with routine mri. Neurology 54(3) (2002) 697-707 